\title{
Reviewers used outside the Editorial Board 2017-2020
}

Liselott Aarsand, Norwegian University of Science and Technology

Sirkka Ahonen, University of Helsinki

Andreas Åkerlund, Södertörn University

Björn Åstrand, Karlstad University

Thom Axelsson, Malmö University

Anne Berg, Uppsala University

Emil Bertilsson, Uppsala University

Ivar Bleiklie, University of Bergen

Hans Bonde, University of Copenhagen

Karl Bruno, Royal Institute of Technology, Stockholm

Mattias Börjesson, University of Gothenburg

John Peter Collett, University of Oslo

Samuel Edquist, Mid Sweden University

Fredrik Eriksson, Swedish Defence University

Trude Evenshaug, University of South Eastern Norway

Anna-Karin Frih, Örebro University

Elin Gardeström, Södertörn University

Mona Gleason, University of British Columbia

Nikolas Glover, Uppsala University

Jákup Reinert Hansen, University of Faroe Islands

Johan Hansson, Umeå University

Harry Haue, University of Southern Denmark

Kim G. Helsvig, Oslo Metropolitan University

Jonny Hjelm, Umeå University

Ann-Kristin Högman, Karlstad University

Hansjörg Hohr, University of Oslo

Marit Hoveid, Norwegian University of Science and Technology

Brit Marie Hovland, NLA University College

Magnus Hultén, Linköping University

Eva Insulander, Stockholm University

Harald Jarning, University of Oslo

Esa-Matti Järvinen, University of Oulu

Thomas Karlsohn, Uppsala University

Kristine Kjærsgaard, University of Southern Denmark

Agneta Knutas, Norwegian University of Science and Technology

Christian Larsen, Danish National Archives

Jesper Eckhardt Larsen, University of Oslo

Kristina Ledman, Umeå University

Agneta Linné, Örebro University

Jens Ljunggren, Stockholm University

Daniel Lövheim, Stockholm University

Lars Lövlie, University of Oslo 
Christian Lundahl, Örebro University

Erland Mårald, Umeå University

Carl Marklund, Södertörn University

Henrik Nordvall, Linköping University

Svante Norrhem, Lund University

Daniel Nyström, Umeå University

Jürgen Oelkers, University of Zurich

Jessica Parland-von Essen, University of Helsinki

Lars Petterson, Dalarna University

Sara Backman Prytz, Stockholm University

Stefan Rimm, Södertörn University

Henrik Román, Uppsala University

Staffan Selander, Stockholm University

Emma Pihl Skoog, Stockholm University

Anna Sténs, Umeå University

Ingerid Straume, University of Oslo

Elisabeth Teige, Volda University College

Fredrik Thue, Oslo Metropolitan University

Aina Tollefsen, Umeå University

Gísli Porsteinsson, University of Iceland

Monika Vinterek, Dalarna University

Arja Virta, University of Turku

Matilda Wiklund, Stockholm University

Ola Winberg, Uppsala University

Thank you very much for your efforts! 